\title{
Every rose has its thorn: Infants' responses to pointed shapes in naturalistic contexts
}

\author{
Aleksandra Włodarczyk ${ }^{\mathrm{a}, *}$, Claudia Elsner $^{\mathrm{a}}$, Alexandra Schmitterer ${ }^{\mathbf{b}}$, Annie E. Wertz ${ }^{\mathrm{a}}$ \\ ${ }^{a}$ Max Planck Institute for Human Development, Max Planck Research Group Naturalistic Social Cognition, Lentzeallee 94, 14195 Berlin, \\ Germany \\ b German Institute for International Educational Research, Schloßstraße 29, 60486 Frankfurt am Main, Germany
}

* Corresponding author at: Max Planck Institute for Human Development, Lentzeallee 94, 14195 Berlin, Germany.

E-mail address: wlodarczyk@mpib-berlin.mpg.de (A. Włodarczyk).

\begin{abstract}
Plants produce dangerous chemical and physical defenses that have shaped the physiology and behavior of the herbivorous predators that feed on them. Here we explore the impact that these plant defenses may have had on humans by testing infants' responses to plants with and without sharp-looking thorns. To do this, we presented 8- to 18-month-olds with plants and control stimuli and measured their initial reaching behavior and subsequent object exploration behavior. Half of the stimuli had sharp-looking thorns or pointed parts while the other half did not. We found that infants exhibited both an initial reluctance to touch and minimized subsequent physical contact with plants compared to other object types. Further, infants treated all plants as potentially dangerous, whether or not they possessed sharp-looking thorns. These results reveal novel dimensions of a behavioral avoidance strategy in infancy that would mitigate potential harm from plants.
\end{abstract}

Keywords: threat; behavioral avoidance; infancy; cognitive development

\section{Introduction}

Plants are dangerous. This fact may seem counterintuitive in the context of WEIRD societies (Western, Educated, Industrialized, Rich, and Democratic; Henrich, Heine, \& Norenzayan, 2010) in which people generally do not have to put a lot of thought into their interactions with plants. Cities and suburbs consist of concrete building and streets, and the people living in them spend significant portions of their lives indoors. Unsurprisingly, under these conditions, human interactions with vegetation can be rare and limited in scope, blinding us to the very real threats that plants can present.

The lives of our hunter-gatherer human ancestors were quite different. The archeological record and studies of modern small-scale societies that rely on hunting and gathering show that plants played a significant role throughout human evolution. Early humans consumed plant foods (Harris \& Hillman, 2014; Henry, Brooks, \& Piperno, 2014; Peters et al., 1981; Ungar \& Sponheimer, 2011) and constructed artifacts from plant materials (Hardy, 2008; Lee, 1993). Modern hunter-gatherer and hunter-horticulturalist societies forage a wide variety of different plant foods from wild environments, specializing in extractive foraging of high energy plant parts (e.g., fruits, tubers, nuts; Cordain et al., 2000; Kaplan, Hill, Lancaster, \& Hurtado, 2000), use plant materials to build tools and shelters (Kortlandt, 1980; Lee, 1993; Sikes, 1994), and extract (often toxic) plant chemicals to aid in hunting and fishing, as well as for medicinal and ritual use (Begossi, Hanazaki, \& Tomashiro, 2002). These interactions are not limited to adulthood. For example, in modern hunter-gatherer societies children as young as 3 to 5 years of age actively engage in foraging activities, particularly with resources that are easy to obtain such as berries and fruits (Crittenden, Conklin-Brittain, Marlowe, Schoeninger, \& Wrangham, 2009; Hawkes, O'Connell, \& Jones, 1995; Greenwald, Eerkens, \& Bartelink, 2016). In these circumstances, humans have frequent and intensive contact with plant life and, as a consequence, regular exposure to the kinds of threats that plants pose. Here we will begin by sketching out two broad categories of plant threat—poisoning and physical injury—and outlining the responses to those threats that have evolved in humans and other animals. Then we will present a novel empirical investigation of a recently-discovered response to plant threat in human infants.

\subsection{Plant threats and protective adaptations in herbivores}

Plants are dangerous because they have evolved a variety of different defenses to protect themselves from the herbivorous predators 
that feed on them. Plants, of course, cannot flee when herbivores begin to attack. Instead they rely on chemical and physical defenses aimed at deterring the damage herbivores can inflict. ${ }^{1}$ An important group of plant defenses are secondary metabolites. All plants produce these chemical compounds, which have a variety of negative effects on herbivores including interfering with their growth, development, and fecundity (Karban \& Baldwin, 1997; Keeler \& Tu, 1983; Palo \& Robbins, 1991). For example, terpenoids are the largest group of plant compounds and can have toxic, inhibitory effects on a wide variety of herbivores (Langenheim, 1994). Terpenoids in conifer resins can trap and drown invading insects, and even remain toxic after evaporation (Keeling \& Bohlmann, 2006). Further, some plant defenses trigger complex behavioral changes in their attackers. For example, tomato plants produce chemicals that induce cannibalism in generalist insects, leading them to consume significantly less plant biomass (Orrock, Connolly, \& Kitchen, 2017).

Herbivores, and omnivores that feed on plants, have evolved countermeasures in response to these toxic chemical compounds. These include detoxification via specific enzymatic pathways and symbiotic relationships with microbes that break down plant toxins (Hagen et al., 2009; Fürstenberg-Hägg, Zagrobelny, \& Bak, 2013), the capacity to detect plant toxins by means of bitter taste receptors (Wiener, Shudler, Levit, \& Niv, 2011), purging the gut with vomiting after ingesting high quantities of toxins (Kingsbury, 1983), and increased nausea during developmental phases that are particularly vulnerable to disruption by toxins (e.g., pregnancy sickness; Profet, 1992). In addition, behavioral avoidance mechanisms like neophobia, and conditioned taste aversions protect animals from ingesting poisonous substances, and may be particularly responsive to plant toxins (Cashdan, 1994, 1998; Lin, Arthurs, \& Reilly, 2017; Reilly, \& Schachtman, 2008; Rioux, Picard, \& Lafraire, 2016). These kinds of mechanisms enable herbivores and omnivores, including humans, to cope with the dangers posed by toxic compounds in plants.

However, toxic chemical substances are not the only dangers that plants pose. Plants also employ physical structures such as thorns ${ }^{2}$ or stinging hairs to deter herbivores. These physical defenses can wound the mouths, digestive systems, and other body parts of large herbivores, leading to chronic mouth injuries and subsequent infestations of parasitic flies (Janzen, 1986). Additionally, thorns may cause septic inflammation themselves by injecting various toxic or pathogenic bacteria into large mammalian herbivores (Halpern, Raats, \& Lev-Yadun, 2007). Herbivores, in turn, have evolved protective countermeasure to avoid contact with thorns. For example, there is evidence that some species of horses, elephants, and tapirs are reluctant to harvest fruits or leaves from thorny plants (Janzen, 1986). Moreover, browsing ungulates modulate their feeding behavior in the presence of thorns by decreasing and slowing down their biting rates (Cooper \& Owen-Smith, 1986; Gowda, 1996).

It is likely that ancestral humans were confronted with thorny plant physical defenses in addition to plant chemical defenses. The natural ecology of hominids likely contained an abundance of thorny vegetation (Bakker et al., 2016). Indeed, archeological evidence suggests thorny plant structures evolved and were present in multiple regions long before the first human populations in those areas (Guo, Sha, Bian, \& Qiu, 2009; Olivier et al., 2009). Without modern medicine or sanitation, injuries from thorny plant structures-similar to other physical injuriescould become quite costly (Gurven, Allen-Arave, Hill, \& Hurtado, 2000; Gurven, Stieglitz, Hooper, Gomes, \& Kaplan, 2012; Sugiyama, 2004). Therefore, recognizing the sharp-angled shape of thorns as dangerous may have been advantageous.

\subsection{Responses to ancestrally-recurrent threats in infancy and early childhood}

A growing body of work provides convergent evidence that infants and young children are sensitive to ancestrally-recurrent naturalistic threats. For example, children as young as three years of age more quickly detect the presence of snakes and spiders compared to control stimuli (LoBue \& DeLoache, 2008; LoBue, 2010a); an advantage that persists into adulthood (New \& German, 2015; Öhman, Flykt, \& Esteves, 2001). Similar results have been found with infants. For example, 8- and 14-month-olds turn more quickly to look at snakes compared to flowers when presented with pairs of stimulus images side by side (LoBue \& DeLoache, 2010), and 5-month-olds look longer in a preferential looking paradigm at schematic spider images than at scrambled versions of the same stimuli (Rakison \& Derringer, 2008). This propensity to quickly orient toward ancestrally-recurrent threatening stimuli appears to play an important role in facilitating learning about danger (LoBue, Rakison, \& DeLoache, 2010), and prepared learning about dangerous animals has been shown in young children across cultures (Barrett \& Broesch, 2012; Barrett, Peterson, \& Frankenhuis, 2016; Broesch, Barrett, \& Henrich, 2014).

In addition to facilitated detection of and learning about ancestrally-recurrent threats, research on low-level human visual perception provides evidence that pointy forms may be detected more quickly and, in some cases, avoided. For instance, adults perceive pointy geometrical shapes as more aversive than round shapes in a subjective rating task, and have more difficulty disengaging their visual attention from them (Larson, Aronoff, \& Stearns, 2007). Further, adults prefer curved contours over sharp angles (Bar \& Neta, 2006; Silvia \& Barona, 2009) — a preference is shared by nonhuman primates (Munar, Gómez-Puerto, Call, \& Nadal, 2015) —and newborn infants preferentially fixate on curved contours compared to sharp-angled ones (Fantz \& Miranda, 1975). This preference applies not only to abstract judgments, but extends to the design of consumer products (Westerman et al., 2012), architecture (Vartanian et al., 2013), and social judgments (Hess, Gryc, \& Hareli, 2013).

However, a systematic avoidance of sharp-angled forms is not found in all circumstances. Some studies show that the preference for curved forms over sharp-angled forms depends on the conditions under which they are presented. Curved forms are preferred when briefly presented but not under free-viewing conditions in humans, but the reverse pattern is found in great apes (Munar et al., 2015), and, notably, an observational study showed that adults walk closer to pointy shaped plants compared to rounded ones (Coss, 2003). Further, experience influences the perception of sharp-angled forms in interesting ways. For example, young children are perceptually sensitive to the presence of pointy-shaped objects (e.g., they more quickly detect syringes in an array of images), but only after they have had a negative experience with the object (e.g., an injection at a doctor's office; LoBue, 2010b).

\subsection{Infants' responses to plant threats}

The existing literature provides evidence that plant threats shaped the physiology and behavior of many animals, including humans, and that human infants are responsive to other ancestrally-recurrent threats. Therefore, the current experiment aims to explore behavioral avoidance strategies that humans use to avoid plant dangers early in ontogeny. Indeed, there is evidence that human infants possess behavioral strategies that protect against plant dangers (Wertz \& Wynn, 2014a). There were two critical aspects of plants that were used to predict the structure of this behavioral avoidance strategy: (1) Plants do not locomote. Therefore, avoiding plant threats can be achieved simply by an infant minimizing their own physical contact with plants. (2)

\footnotetext{
1 Plants have also evolved mechanisms that exploit herbivores to serve their own ends (e.g., seed dispersal). These mechanisms are an integral part of the dynamics between plants and herbivores, but they are beyond the scope of the current paper.

2 English botanical usage distinguishes between a prickle (a pointy emergence from the cortex), a spine (a pointy part of a leaf), and a thorn (a pointy branch). For the sake of clarity, we are referring to these categories with the single word "thorns" throughout this paper.
} 


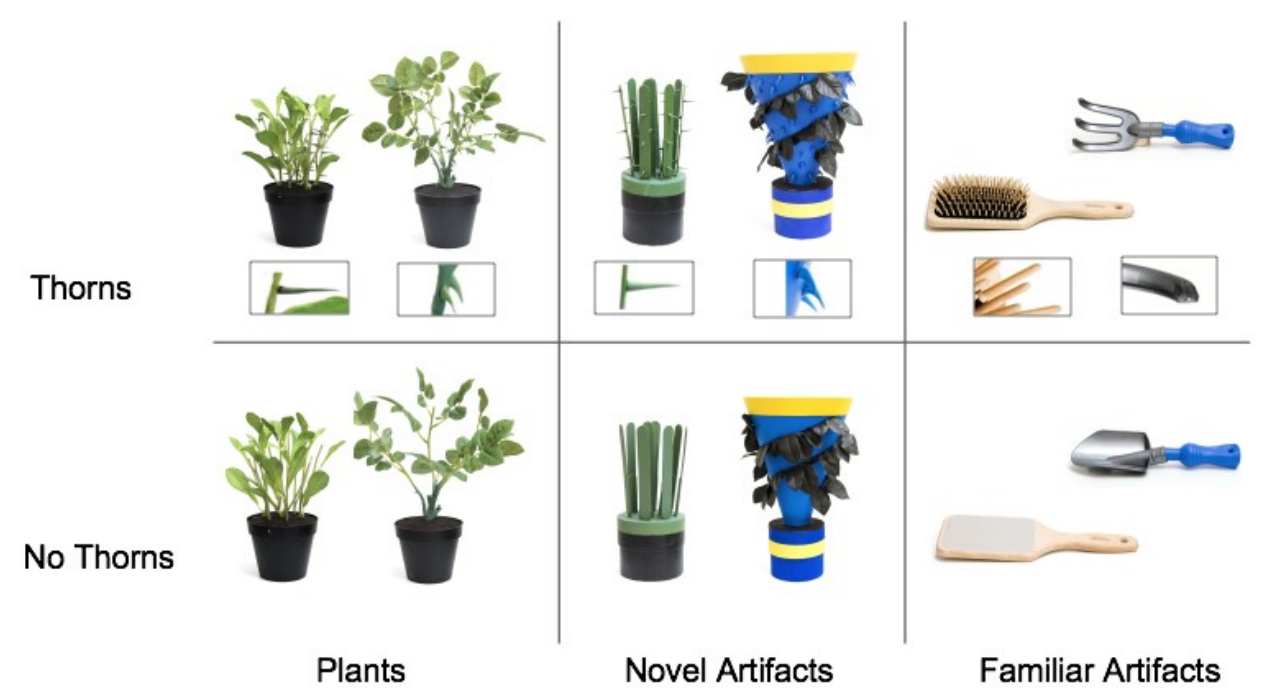

Figure 1. Stimulus set used in the experiment. The stimuli depicted in the top row have thorns (plants and novel artifacts) or pointed parts (familiar artifacts). The stimuli depicted in the bottom row are matched to those on the top row but have no thorns or pointed parts; close-up insets depict the thorn or pointed part shapes used on each thorny object.

Because plants are in coevolutionary relationships with many different species, primarily insect species that have very different sensory systems and physiologies than humans, plants do not have visual properties that reliably signal human-relevant toxicity (Keeler \&Tu, 1983; Palo \& Robins, 1991). Therefore, the best strategy, especially in periods of vulnerability like early ontogeny, is to minimizing physical contact with all unknown plants, regardless of how they look, until one has some additional information about their properties.

To test this, Wertz \& Wynn (2014a) presented 8- to 18-month-olds with benign-looking plants (e.g., parsley), novel manmade artifacts matched to shape and color features of the plants, familiar artifacts (e.g., a small lamp), and natural objects (e.g., shells) and their reaching behavior was assessed. As predicted, infants exhibited a striking reluctance to touch plants compared to the other types of entities. Avoiding physical contact with plants in this way would protect infants from the dangers posed by plants' chemical and physical defenses. Moreover, separate studies showed that infants use social information to guide their interactions with plants (e.g., to learn which plants can be eaten; Wertz \& Wynn, 2014b).

\subsection{The current study}

The previous study investigated one aspect of infants' behavioral avoidance strategy toward plants: namely, the initial reluctance to touch benign-looking plants (Wertz \& Wynn, 2014a). Here we explore two novel aspects of the proposed strategy. First, we test whether infants' behavioral avoidance strategy extends to their manual exploration of plants after they make contact with them. If so, in addition to the previously-discovered reluctance to touch plants, this predicts that infants should spend less time in physical contact with plants once they first touch them and touch plants less frequently than other types of entities. Second, we examine whether infants respond differently to observable physical plant threats-visibly pointy thorns-compared to benign-looking plants that could nevertheless contain unobservable toxic chemical defenses. One possibility is that infants treat observable and unobservable plant threats the same since both types of threats have been present across evolutionary time, and avoiding physical contact would effectively mitigates both types. Alternately, because there is less uncertainty about the presence of physically observable plant threats like thorns compared to unobservable toxic chemical compounds, infants may show increased behavioral avoidance of plants when they possess sharp-looking thorns.

To test these questions, we used a paradigm adapted from Wertz \& Wynn (2014a) in which 8- to 18-month-old infants were presented with three categories of stimulus objects: plants, novel artifacts matched to shape and color features of the plants, and familiar artifacts. Half of the stimulus objects in each category had thorns or pointed parts while the other half did not. We assessed infants' initial reaching behavior toward, and subsequent manual exploration of, each stimulus object.

\section{Materials and Methods}

\subsection{Participants}

Participants were healthy, full term 8- to 18-month-old infants ( $N=42,19$ females, $M_{\text {age }}=13$ months, 5 days; range $\left.=7 ; 30-17 ; 27\right)$ recruited from a large city in Germany. This age range and sample size were based on Wertz \& Wynn (2014a). The study was approved by the responsible Ethics Committee and parents gave written consent for their child's participation. Families were compensated with $€ 10$ and infants were given a participation certificate.

\subsection{Stimuli}

The stimulus set consisted of three types of objects: realistic-looking artificial plants, novel manmade artifacts, and familiar manmade artifacts. There were four objects of each stimulus type grouped into matched pairs. One stimulus object in each pair had thorns or pointed parts while the other was identical except that it lacked thorns or pointed parts (see Figure 1). The thorns used in our stimulus set had two different shapes: a triangular shape similar to a rose thorn, and a thin needle shape similar to a cactus thorn (see Figure 1 insets). The thorns on the plants and novel artifacts were made out of a soft child-safe modeling clay to ensure that children could not be injured. The familiar objects had child-safe pointed parts that matched the two thorn shapes used on the plants and novel artifacts.

The stimuli plants were four realistic-looking artificial plants of two different types, made out of plastic stems and fabric leaves arranged in black plastic pots, purchased from a large chain store (see left column of Figure 1). One of the plants of each type had thorns glued to the stems and branches while the other did not. We used only realistic-looking artificial plants in this stimulus set so that infants were not exposed to potentially harmful thorns on real plants, however previous studies have shown that infants of this age respond similarly to real and artificial plants (Elsner \& Wertz, under revised review; Wertz \& Wynn, 
There were two types of novel artifacts designed to match shape and color features of the stimulus plants (see middle column of Figure 1). The first type of novel artifact matched the size and green color of one of the plant types. It was made from a cylinder-shaped green and black cardboard box base with green popsicle sticks protruding from the top; one of these green novel artifacts had cactus-like green thorns. The second type of novel artifact matched the size and leaf shape of the other plant type. It was made from a blue and yellow cardboard cone arranged on top of a blue and yellow cylinder-shaped cardboard box base, and wrapped with black leaves of the exact shape used on one type of stimulus plant; one of these blue novel artifacts had blue curved downward rose-like thorns.

The familiar artifacts were included in the stimulus set in order to examine whether infants respond differently to broader classes of familiar compared to unfamiliar objects. The plant stimuli would be relatively more familiar to infants than our novel artifact stimuli. Therefore, we included exemplars of artifacts that would also be relatively more familiar to infants. There were two pairs of familiar artifacts, with one object in each pair containing thorn-like shapes (see right column of Figure 1). The first pair included a wooden hairbrush and a wooden hand mirror, and the second pair consisted of a children's plastic rake and plastic shovel.

See Supplementary Information (SI) Sections 1.1.-1.3. for further details of the stimuli.

The focus of our investigation was infants' behavior towards plants and their thorns in particular, not towards the pots in which the plants were presented. Therefore, we treated the top parts of all stimulus objects as our area of interest. The "top parts" of the stimuli were defined as (i) the stems, leaves, and thorns (when applicable) of the plants, (ii) the cones, fabric leaves, popsicle sticks, and thorns (when applicable) of the novel artifacts, and (iii) the flat, wide (i.e., non-handle) parts of the familiar artifacts including the pointed parts (when applicable; see Figure 1 and SI Section 2 for further details).

\subsection{Procedure}

Infants sat on their parents' laps on the opposite side of the table from the experimenter approximately $145 \mathrm{~cm}$ away. The experimenter placed the twelve stimulus objects one at a time approximately $25 \mathrm{~cm}$ away from the infant, while saying in German, "Look, what I've got!" "'Schau mal, was ich hier habe!"; see Video S1 and Video S2). The experimenter touched only the bottom parts of the objects throughout the session (i.e., the plant pots, the bases of the novel artifacts, and the handles of the familiar artifacts) so as not to cue infants' touching behavior toward the top parts of the stimulus objects. Parents were instructed to keep their eyes closed during the stimulus presentation. The experimenter looked down at the table and maintained a neutral facial expression while each stimulus object was in front of the infant. Each stimulus object remained in front of the infant until (i) 5 seconds elapsed after the infant first touched the top part of the object, (ii) 30 seconds elapsed in the case where the infant touched only the bottom part of the object and failed to touch any other part or (iii) 30 seconds elapsed without a touch. When one of these three criteria were met, the trial was ended.

The order of presentation of the stimulus objects was blocked into thorny and non-thorny stimulus objects and randomized within each block. That is, the six objects with thorns were presented serially in one block and the six objects without thorns were presented serially in another block. Whether infants were presented with thorny or non-thorny objects first was counterbalanced across participants. The order of presentation of the six objects in the first block of the presentation was randomized, and then adjustments were made to ensure that each object type (plant, novel artifact, familiar artifact) appeared first roughly equally across participants. Complete randomization across all the objects within each block was not possible due to the number of presented stimuli. The order of presentation in the second block was the mirror image of the presentation order of the second block. Additionally, the side on which the handle of the familiar artifacts was presented (left vs. right) was counterbalanced across those stimulus objects within each block.

After the object presentation phase concluded, parents completed a battery of questionnaires (see Section 2.5 below).

\subsection{Coding infant behavior}

\subsubsection{Video recording}

Infants' behavior during the session was recorded on video with four GoPro Hero 4, Black Edition cameras. Each session was recorded from four different angles: a front-view of the infant and the stimulus object, a side-view of the experimenter placing each object in front of the infant, as well as two close up side views (i.e., left and right) of the object and infants' hands. Recorded videos were imported into ELAN, a program for annotating multi-media recordings (v.4.9.1; Lausberg \& Sloetjes, 2009) for subsequent behavioral coding.

\subsubsection{Coding procedure}

Infants' touch behavior toward to the top part of the stimulus object was coded during each trial. Each trial began when the experimenter released her hands from the stimulus object after placing it on the table in front of the infant (as in Elsner \& Wertz, under revised review; Wertz \& Wynn, 2014a) and ended as outlined in Section 2.3. A "touch" was coded as any part of the infant's hand coming into contact with the stimulus object. Eight individual trials (out of the total 504 trials) were excluded from further analysis due to procedure error (two trials), failure to present a stimulus object by the experimenter (one trial), infants throwing a stimulus object on the ground (three trials), and infants leaving their mothers' laps (two trials).

\subsubsection{Coding infants' touch behavior}

Three aspects of infants' touch behavior to the top parts of the stimulus object were coded during each trial: (i) Latency: the elapsed time before infants first made contact with the stimulus object; if infants did not touch the object during the trial, this was coded as the maximum trial length of 30 seconds, (ii) First Touch Duration: the duration of infants' first touch of the stimulus object, (iii) Touch Frequency (thorny stimuli only): how frequently infants touched the different parts of the stimulus object in the 5 second window after their initial contact with it (i.e., number of touches to thorny areas, non-thorny areas, and both types of areas simultaneously).

Latency and First Touch Duration were initially coded for infants' first touch to the top part of all of the stimulus objects regardless of whether that touch contacted a thorny or non-thorny part of that object. Subsequently, infants' Latency and First Touch Duration were coded separately for first touches to the thorny and non-thorny parts of the stimulus objects (e.g., latency to the first touch of a thorny area, and latency to the first touch of a non-thorny area). Touch Frequency was coded only for the thorny stimulus objects because these were the only stimulus objects with separate thorny and non-thorny parts.

\subsubsection{Interrater reliability}

A randomly selected $25 \%$ of the videos were coded by a second independent coder. Coder agreement was high for all dependent variables: Latency (Pearson's correlation $r=.97$ ), First Touch Duration (Pearson's correlation $r=.94)$, and Touch Frequency $(\mathrm{k}=.91)$.

\subsubsection{Coding experimenter behavior}

In order to assess whether the experimenter's behavior during the object presentation phase may have inadvertently influenced infants, a randomly selected third of the videos were coded by two independent coders who rated the experimenter's behavior during each trial. The videos were first edited to remove the portion that showed the stimulus 
object so that the coders were blind to trial type. Based on the experimenter's facial expressions and movements the coders rated the expected object type that was presented (plant vs. non-plant). A binomial test indicated that the proportion of correctly rated objects was not significantly different from chance (Coder $1=51 \%$, Coder $2=60 \%$, chance level of $56 \%$ given the proportion of plant to non-plant objects in the stimulus set; p's > .05 for both coders). In addition, the agreement between the two coders was very low for the presented objects (Cohen's $\mathrm{K}=-.10)$ suggesting that the experimenter did not systematically give cues to participants that could systematically affect their responses.

\subsection{Parent questionnaires}

After the presentation phase of the study, parents completed questionnaires assessing their infants' prior experience with plants generally (Elsner \& Wertz, under revised review; Wertz \& Wynn, 2014a; 2014b), thorny plants specifically, and the familiar artifacts used in the study. The set of questions related to infants' general experience with plants was assessed separately for indoor and outdoor plants as well as for two time points (last summer vs. last several months). Parents also completed questionnaires assessing their own knowledge about plants, as well as 5 subscales of an infant temperament questionnaire (IBQ-R; Gartstein \& Rothbart, 2003). The order of the questionnaires was randomized across participants (See Supplementary Section 6 for further details).

\section{Results}

In order to test whether infants exhibit a behavioral avoidance strategy for plants, and examine whether such a strategy is influenced by the presence of thorns, we assessed infants' initial reaching for and subsequent manual exploration of the presented stimulus objects. Descriptive statistics for our outcome variables, Latency, First Touch Duration, and Touch Frequency, are provided in Table 1. Preliminary analyses showed that there were no main effects of block order, except in the case of the touch frequency analysis. The analyses were performed with $\mathrm{R}$ ( $\mathrm{R}$ version 3.3.2; $\mathrm{R}$ Core-Team, 2016). In all analyses, we used a linear mixed-effects approach (Baayen, Davidson, \& Bates, 2008; Bates, Mächler, Bolker, \& Walke, 2014).

\subsection{Latency}

In order to test the prediction that infants would take longer to touch plants compared to other objects, we assessed the effect of Object Type on the time elapsed before infants first made contact with the different types of stimulus objects (Latency; see Table 1 for the descriptive statistics). Latency values were log transformed to correct for non-normality and analyzed with a linear mixed-effects model using a Gaussian distribution (Imer function from R-package Ime4; Bates et al., 2014). In the model, participants were treated as random effects, whereas Object Type (Plants, Novel Artifacts, Familiar Artifacts) and Thorniness (Thorny vs. Non-thorny Objects) as well as their interaction served as within participant fixed-effects. In addition, Age was included in the models as a control variable to examine whether infants responded differently across our 8- to 18-month-old age range. Omnibus effects were calculated based on Type II model comparisons (using the ANOVA function in the R-package car; Fox \& Weisberg, 2011). Post hoc analyses were carried out using single-degree-of-freedom contrasts based on the cell mean estimates in separate models with the same parameters ( $g$ lth function from R-package multicomp; Hothorn, Bretz, \& Westfall, 2008).

Main and interaction effects are presented in Table 2. The results showed a significant main effect of Object Type. As predicted, infants took significantly longer to touch plants than other object types. The latency for plants was significantly longer than the latency for both novel artifacts $(t=5.14, p<.001)$ and familiar artifacts $(t=3.16, p<.01$; see Figure 2$)$. In addition, there was a trend that the latency for familiar objects was longer than the latency for novel artifacts $(t=1.96, p=.05)$. There was no main effect of Thorniness nor was there a Thorniness by Object Type interaction. There was also no main effect of infant age.

In addition, consistent with the prediction that infants minimize physical contact with plants, a nonparametric Friedman's ANOVA indicated that the number of trials without a touch was significantly affected by Object Type $(x 2(2)=14.11, p<.01)$. The percentage of "notouch" trials per object type were as follows: $25 \%$ for novel artifacts, $20 \%$ for familiar artifacts, and $42 \%$ for plants. Wilcoxon sign-ranked tests showed that there were significantly more no-touch trials for plants $(\mathrm{Mdn}=1.5)$ compared to novel artifacts $(\mathrm{Mdn}=0 ; \mathrm{Z}=-3.49, p<.001, r=-$ .38 ) and familiar artifacts ( $\mathrm{Mdn}=0 ; \mathrm{Z}=-3.28, p<.01, r=-.36)$, but there was no difference between the amount of no-touch trials for novel artifacts $(\mathrm{Mdn}=0)$ and familiar artifacts ( $\mathrm{Mdn}=0 ; Z=-.96, p>.05, r=-.10)$. Because our main prediction was that infants would avoid touching plants we anticipated that there would be no-touch trials and therefore included these no-touch trials in the linear mixed effects model above. However, analyzing the latency data excluding the no-touch trials yielded a similar pattern of results (see SI section 4 for further details).

These results show that as predicted, infants take longer until they make contact with plants compared to both novel artifacts and familiar artifacts, replicating previous results (Wertz \& Wynn, 2014a). However, the presence of thorns did not significantly impact the timing of infants'

Table 1

Descriptive statistics for latency, first touch duration, and touch frequency

\begin{tabular}{|c|c|c|c|c|c|c|c|}
\hline & \multicolumn{2}{|c|}{ Latency } & \multicolumn{2}{|c|}{ First Touch Duration } & \multicolumn{3}{|c|}{$\begin{array}{c}\text { Touch Frequency } \\
\text { (Thorny Objects Only) }\end{array}$} \\
\hline & Thorny Objects & $\begin{array}{c}\text { Non-thorny } \\
\text { Objects }\end{array}$ & $\begin{array}{l}\text { Thorny } \\
\text { Objects }\end{array}$ & $\begin{array}{l}\text { Non-thorny } \\
\text { Objects }\end{array}$ & $\begin{array}{l}\text { Thorny } \\
\text { Parts }\end{array}$ & $\begin{array}{l}\text { Both } \\
\text { Parts }\end{array}$ & $\begin{array}{c}\text { Non-thorny } \\
\text { Parts }\end{array}$ \\
\hline $\begin{array}{l}\text { Plants } \\
\text { Novel }\end{array}$ & $13649(3861)$ & $9628(2723)$ & 705 (166) & $666(157)$ & $0.3(0.1)$ & $0.4(0.2)$ & $0.9(0.3)$ \\
\hline $\begin{array}{l}\text { Artifacts } \\
\text { Familiar }\end{array}$ & $3684(1042)$ & 4062 (1149) & 564 (115) & $1854(409)$ & $0.8(0.3)$ & $1.2(0.3)$ & $2.2(0.5)$ \\
\hline Artifacts & $5371(1519)$ & 6407 (1833) & 953 (194) & $1554(331)$ & $1.8(0.4)$ & $0.8(0.2)$ & $1.1(0.3)$ \\
\hline
\end{tabular}

Note. Standard errors are provided in parentheses.

Table 2

ANOVA results for linear mixed-effect models for the latency and first touch duration.

\begin{tabular}{|c|c|c|c|c|}
\hline \multirow[t]{2}{*}{ Effect } & \multicolumn{2}{|c|}{ Latency } & \multicolumn{2}{|c|}{ First Touch Duration } \\
\hline & $\mathrm{F}$ (df, dfres) & $p$ & $\mathrm{~F}$ (df, dfres) & $p$ \\
\hline Thorniness & $0.02(1,204)$ & $>.05$ & $14.33(1,167)$ & $<.001^{* \star \star}$ \\
\hline Object Type & $13.43(2,204)$ & $<.001^{* * *}$ & $4.63(2,165)$ & $<.05^{\star}$ \\
\hline Age & $1.11(1,40)$ & $>.05$ & $0.003(1,37)$ & $>.05$ \\
\hline Thorniness $x$ Object Type & $0.89(2,204)$ & $>.05$ & $5.39(2,162)$ & $<.01^{* *}$ \\
\hline
\end{tabular}

Note. F values (latency and first touch duration) for effects using Type II Wald F tests with Kenward-Roger df. Asterisks indicate significant results. 


\section{Latency}

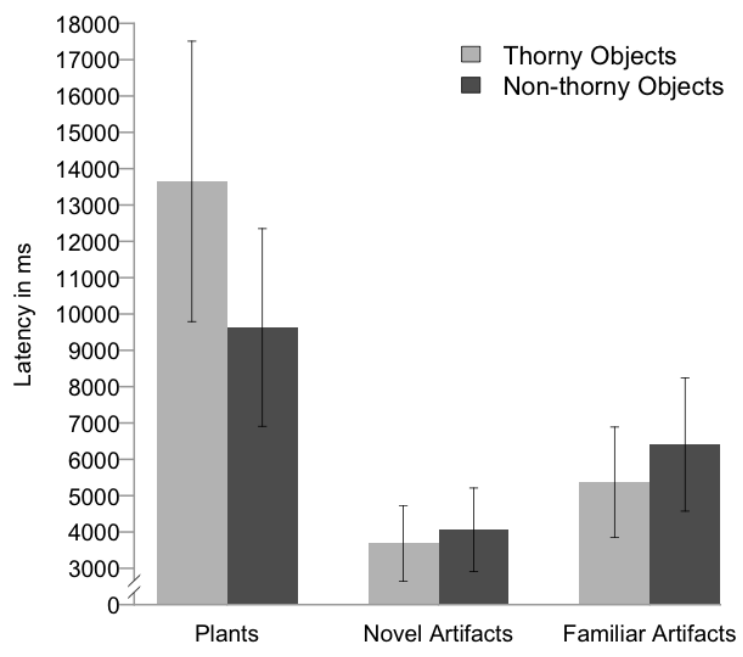

Object Type

Figure 2. Back-transformed mean latency until the first touch to any top part of the different object types. Error bars represent standard errors.

initial reach for any of our stimulus objects, including the plants.

\subsection{First touch duration}

Next, in order to examine whether infants showed minimized subsequent physical contact with plants in addition to an initial reluctance to touch, we analyzed the effects of Object Type and Thorniness on the duration of infants' first touch to the top part of the objects (i.e., First Touch Duration). In this analysis, we were interested in the duration of the touches that occurred, therefore we excluded trials without a touch from the analysis. Again, raw durations were log transformed and analyzed with a linear mixed-effects model using a Gaussian distribution. Infant participants were modeled as random effects, whereas Object Type (Plants, Novel Artifacts, Familiar Artifacts) and Thorniness (Thorny vs. Non-thorny Objects) as well as their interaction were treated as within participant fixed-effects. We also used Age as a control variable.

Main and interaction effects are presented in Table 2. The results showed a significant main effect of Object Type and Thorniness, as well as a significant interaction effect for Object Type and Thorniness. As in the latency analyses, there was no effect of infant age. Consistent with the prediction that infants minimize subsequent physical contact with plants, infants spent less time in contact with plants compared to nonplant objects. Post-hoc cell-mean comparisons revealed that First Touch Duration was shorter for plants compared to both novel artifacts $(t=$ $2.08, p<.05)$ and familiar artifacts $(t=3.01, p<.01$; see Figure 3$)$.

Moreover, infants spent less time in contact with the thorny objects than the non-thorny objects $(t=3.52, p<.001)$. However, the interaction effect of Object Type and Thorniness was explained by different effects of thorniness across the object types. Thorniness affected infants' behavior towards artifacts but not plants. Infants spent significantly less time touching thorny novel artifacts than non-thorny novel artifacts $(t=4.63, p<.001)$. The same trend was found for familiar artifacts $(t=1.93, p=.05)$. Yet, the duration of the first touch did not differ significantly between thorny and non-thorny plants $(t=0.20, p>.05)$, mirroring the results from the latency analysis indicating that infants treat all plants as potentially dangerous.

Follow-up comparisons further support this interpretation. Although infants spent less time in contact with thorny objects than with non-thorny objects, we did not find differences in First Touch Duration between thorny plants compared to both thorny novel and thorny familiar artifacts ( $p$ 's >.05); there was a difference between thorny novel and thorny familiar artifacts $(t=2.12, p<.05)$. However, for the non-thorny objects, First Touch Duration was shorter for plants compared to novel artifacts $(t=3.72, p<.001)$ and familiar objects $(t=3.12, p<.01)$, but First Touch Duration did not differ between novel and familiar artifacts $(t=.67, p>.05)$.

Taken together, these results are consistent with the prediction that infants minimize their subsequent physical contact with plants compared to novel and familiar artifacts. Moreover, although infants spend less time touching thorny artifacts, they treat thorny and non-thorny plants the same.

\subsection{Touch frequency to specific parts of the objects (thorny objects only)}

To investigate the effect of thorns on infants' subsequent manual exploration in more detail, we assessed the effect of Object Type on touch frequency to specific parts of the thorny objects (i.e., the number of touches to thorny parts, non-thorny parts, and touches that contact both parts at the same time; see Figure 4). The data were analyzed using generalized linear models with a Poisson distribution and a correction for overdispersion due to zero-inflation (g/mer function from R-package Ime4; Bates et al., 2014). In the model, infant participants and an individual variable correcting for over dispersion were treated as random effects, whereas Object Type (Plants, Novel Artifacts, Familiar Artifacts) and Place of Touch (Thorny, Non-thorny, Both) as within participant fixed-effects. In addition, Age and Block Order was included as a control variable.

Main and interaction effects are presented in Table 3. The results showed a main effect of Object Type, and a main effect of Place of Touch, as well as an interaction effect of Object Type and Place of Touch. Overall, consistent with the prediction that infants minimize physical contact with plants, infants touched plants less frequently 


\section{First Touch Duration}

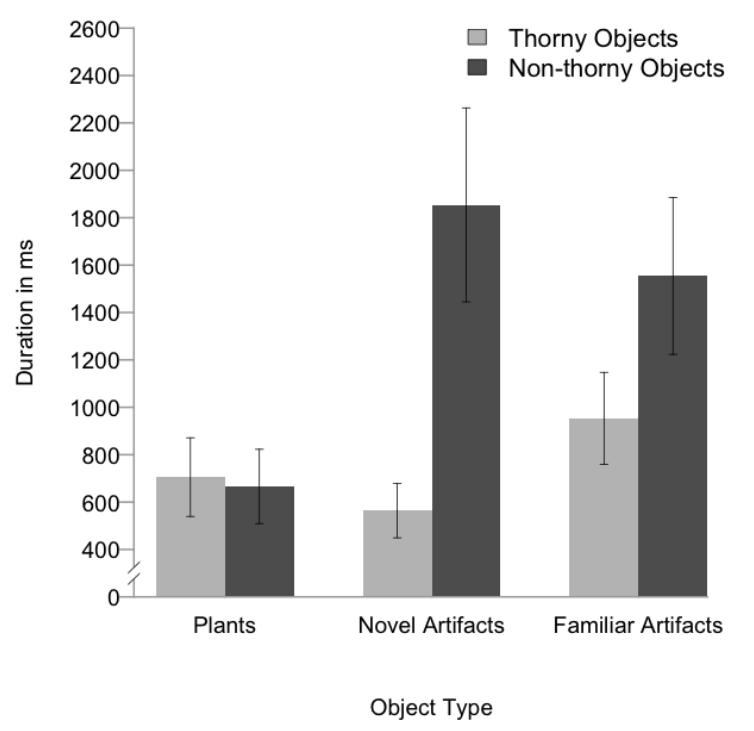

Figure 3. Back-transformed mean first touch duration for the different object types. Error bars represent standard errors.

Table 3

ANOVA results for generalized linear mixed-effects models for touch frequency.

\begin{tabular}{|c|c|c|}
\hline \multirow[t]{2}{*}{ Effect } & \multicolumn{2}{|c|}{ Frequency } \\
\hline & $\mathrm{X} 2(\mathrm{df})$ & $p$ \\
\hline Place of Touch & 19.62(2) & $<.001^{* * *}$ \\
\hline $\begin{array}{l}\text { Object Type } \\
\text { Age }\end{array}$ & $\begin{array}{c}42.09(2) \\
0.01(1)\end{array}$ & $\begin{array}{l}<.001^{* * *} \\
>.05\end{array}$ \\
\hline Order & $5.10(1)$ & $<.05^{\star}$ \\
\hline Place of Touch $x$ Object Type & $33.84(4)$ & $<.001^{* * *}$ \\
\hline
\end{tabular}

Note. $\mathrm{X} 2$ values (frequency) for effects using Type II Wald F tests with Kenward-Roger df. Asterisks indicate significant results.

compared to both novel artifacts $(t=5.89, p<.001)$ and familiar artifacts $(t=5.007, p<.001$; see Figure 5). There was no difference in amount of touches between the non-plant objects $(t=1.42, p>.05)$. As in the previous analyses, there was no effect of age. However, unlike the other analyses, we found a main effect of presentation order (thorny objects presented first vs. non-thorny objects presented first). Infants touched the stimuli more frequently when non-thorny objects were presented first compared to when thorny objects were presented first $(t=2.90, p<.01)$.

Next, we directly compared infants' touch frequencies to specific object parts. Infants touched thorny parts of plants less frequently than the thorny parts of novel artifacts $(t=3.20, p<.01)$ and familiar artifacts $(t=4.50, p<.001)$. Interestingly, infants touched the thorny parts of familiar artifacts more often compared to novel artifacts $(t=2.93, p<.01)$, indicating that they do not systematically avoid thorny shapes in all contexts. The results were similar for infants' touches that contact both thorny and non-thorny parts simultaneously (hereafter "both" touches). Infants touched both parts of plants less frequently than both parts of novel artifacts $(t=4.11, p<.001)$ and familiar objects $(t=2.52, p<.05)$. However, there was no difference in the amount of both touches to novel and familiar artifacts $(t=1.88, p=.10)$. Finally, infants touched the non-thorny parts of plants $(t=4.24, p<.001)$ and familiar artifacts $(t=3.76, p<.001)$ less often than novel artifacts. There was no difference in touch frequency for the non-thorny parts of plants and familiar artifacts $(t=0.56, p=0.60)$.

These results provide additional support for the prediction that infants minimize subsequent physical contact with plants: Infants touched plants less frequently overall than other object types. The comparisons of touches to specific parts of the thorny objects suggest that infants particularly avoid touching thorny parts of plants compared to the thorny parts of novel and familiar artifacts.

Given that we found the first hint that infants particularly avoid touching the thorny parts of plants, we decided to do a more fine-

A.

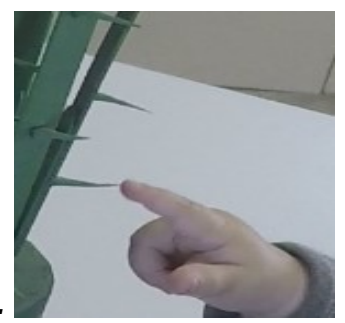

B.

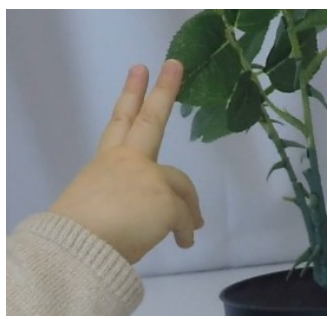

C.

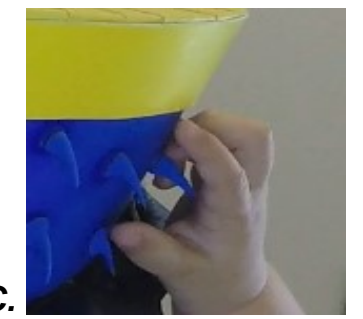

Figure 4. Different types of touches to the thorny objects included in the touch frequency analysis. These were touches that contacted thorny parts (Panel $A$ ) non-thorny parts (Panel $B$ ), and both parts at the same time (Panel $C$ ). 
Touch Frequency to Specific Parts of Objects

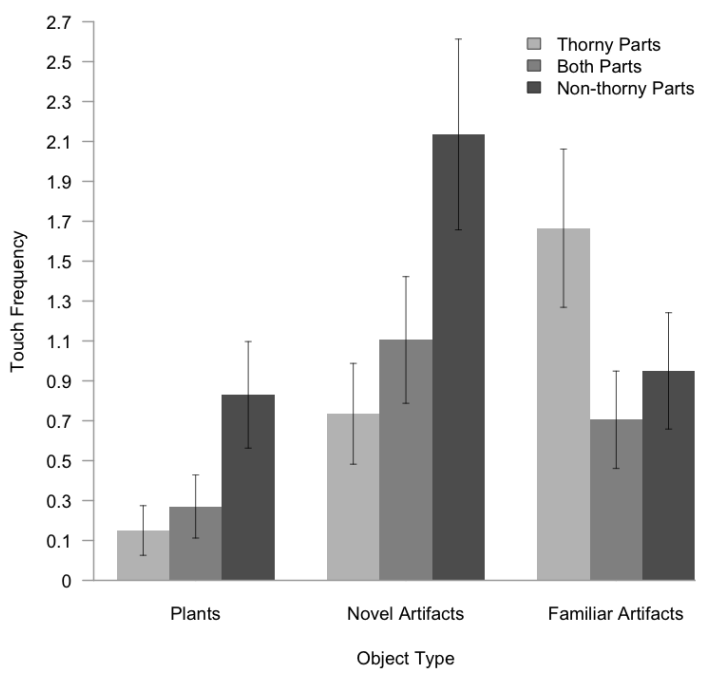

Figure 5. Back-transformed touch frequency to specific parts of the objects (thorny objects only). Error bars represent standard errors.

grained analysis of how the location of infants' touch impacts the latency and the first touch duration. We provide a brief summary here and report the full results in Supplementary Section 5. When analyzing the latency until infants' first touch to specific parts of the objects (i.e., thorny parts of the thorny objects, non-thorny parts of the thorny objects, and the non-thorny objects), the results showed additional evidence of the impact of thorns on infants' behavior. Infants' took longer to touch the thorny and non-thorny parts of the thorny plants compared to their first touch of the non-thorny plants. Infants showed a similar pattern of response for novel artifacts, even though they took longer overall to touch plants. However, infants reached more quickly for the thorny parts of familiar artifacts, suggesting that infants do not avoid thorns in all contexts.

Similarly, when analyzing the duration of infants' first touch to specific parts of the objects, infants did not differ in the amount of time they remained in contact with the different parts of plants after first touching them. In contrast, infants spent less time in contact with thorny novel artifacts compared to non-thorny ones, but spent more time touching the thorny parts of the thorny familiar artifacts than the non-thorny parts. In fact, infants spent the same amount of time in contact with the thorny parts of familiar artifacts after first touching them as the nonthorny familiar artifacts (see Supplementary Section 5 for further details).

\subsection{Questionnaire analyses}

These analyses assessed whether infants' prior experiences influenced their behavior toward our stimulus objects. We were particularly interested in infants' prior experience with plants generally, thorny plants specifically, and the familiar artifacts used in the study. Additionally, we assessed parents' self-reported knowledge about plants, as well as an infant temperament (IBQ-R; Gartstein \& Rothbart, 2003) in relation to infants' responses.

Correlational analyses revealed that most of the questionnaire measures used in the study were not systematically related to Latency, First Touch Duration, and Touch Frequency (see Supplementary Section 6 for further details of the questionnaire analyses). Among a small number of significant correlations which held after the correction for multiple comparisons, the majority were related to infants' behavior towards novel and familiar artifacts, but not plants (e.g., infants who scored higher on the Approach subscale of the temperament questionnaire had shorter latencies to touch familiar artifacts; see Supplementary Section 6 for further details).

Importantly, our questionnaires asked about how often parents stopped their infant from touching plants. We asked this question about plants generally and thorny plants specifically. Almost none of the correlations between parental prohibition and infants' behavior towards plants (or any of the other stimulus objects) were significant. The only significant correlations between parental prohibition and infants' behavior towards plants which persisted after the correction for multiple comparisons $(p$-value $<.0042)$ were the correlation between parental prohibition of touching outdoor plants in the last summer months and touch frequency for the non-thorny parts of thorny plants $(r=-.60, p=.003)$, as well as the correlation between parental prohibition of touching outdoor plants in the last several months and the touch frequency for the non-thorny parts of thorny plants $(r=-.67, p=.0005)$. Additionally, there was a significant correlation between parental prohibition of touching outdoor plants in the last summer months and infants' touch frequency for the non-thorny parts of thorny familiar artifacts $(r=.57, p=.004)$.

Further, although the correlations did not persist through the corrections for multiple comparisons, a hint of a relationship was found in positive correlations between parental prohibition of touching plants generally and infants' latency to touch thorny plants (prohibit touching indoor plants in the last several months $r=.34, p=.03$; prohibit touching outdoor plants in the last several months $r=.36, p=.02$; prohibit touching outdoor plants in the last summer months $r=.33, p=.03$ ), but there was no correlation with infants' latency to touch non-thorny plants (all $p$ 's > .05). However, it is important to keep in mind that these correlations did not persist after the correction for multiple testing, and we found no correlation between parental prohibition to touch thorny plants specifically and infants' latency to touch thorny plants or infants' touch frequency for non-thorny as well as thorny plants (all p's > .05).

In summary, we found some evidence that prohibition experience influences infants' subsequent manual exploration of thorny plants-although the correlations only held for the non-thorny parts of these thorny plants. The more often infants were prohibited from touching outdoor plants in general the less frequently they touched non-thorny parts of thorny plants. We also found the hints that parental prohibition may be associated with increased reluctance to touch thorny plants. However, these relationships were weak and there is no evidence that prohibition experience influences infants' behavior toward non-thorny plants. This lack of association with non-thorny plants is consistent with previous datasets that also looked for, but failed to find a relationship between parental prohibition and infants' initial reaching behavior towards benign-looking plants (Elsner \& Wertz, under revised review; Wertz \& Wynn, 2014a).

\section{Discussion}

The findings presented here provide evidence that 8- to 18- month-old infants possess a behavioral avoidance strategy that minimizes their exposure to plant threats. The results replicate previous findings showing that infants are reluctant to touch plants compared to other types of entities (Elsner \& Wertz, under revised review; Wertz \& Wynn, 2014a) and reveal two important and novel dimensions to this behavioral avoidance strategy. First, the current results show that the behavioral avoidance strategy towards plants in infancy includes both an initial reluctance to touch and a minimization of subsequent physical contact with plants. Specifically, infants spend less time in contact with plants after first touching them and touch plants less frequently than other types of entities. Second, infants treat all plants as potentially dangerous, whether or not the plant possesses observable threats (i.e., thorns). 


\subsection{Implications for the design of human adaptations to mitigate plant dangers}

The current findings add to a growing body of literature on responses to ancestrally-recurrent threats in infancy and early childhood (e.g., Barrett \& Broesch, 2012; Barrett et al., 2016; LoBue \& DeLoache, 2008; LoBue, 2010a; Rakison \& Derringer, 2008) and support our argument that the structure of the behavioral avoidance strategy is designed around the fact that plants are rooted to the ground and therefore cannot inflict costs unless they are approached. Avoiding contact with plants in the way we have demonstrated here would be an effective protective strategy because it reduces the risk of poisoning and physical injury from chemical and physical plant defenses. This type of strategy operates alongside other protective mechanisms (e.g., enzymatic detoxification pathways, Hagen et al., 2009; neophobia, Rioux at al., 2016; pregnancy sickness, Profet, 1992) to mitigate the dangers that plants pose.

Prior to running the current experiment, it was unclear whether the reluctance infants showed towards touching benign-looking plants found in previous studies (e.g., Wertz \& Wynn, 2014a) would become more pronounced for plants that possessed observable visible indicators of threat (i.e., sharp-looking thorns). Our results indicate that visible indicators of plant threat in fact do not significantly increase infants' reluctance to touch plants nor significantly reduce the duration of infants' initial physical contact with plants. This suggests that the structure of the behavioral avoidance strategy in infancy is to initially treat all plants as potentially dangerous-whether they are benign-looking or covered in thorns. We argue that this design likely reflects the fact that plants (i) manufacture a variety of chemical defenses against herbivores, but (ii) do not reliably signal human-relevant toxicity via predictive color patterns or shapes (Keeler \& Tu, 1983; Palo \& Robbins, 1991). Therefore, the visual appearance of a plant is not a good indicator of whether or not it is dangerous.

While infants treat plants with and without thorns similarly, our results show that 8- to 18-month-olds nevertheless do appear to be sensitive to the potential harm that pointed thorns can inflict. Interestingly, this is evident at the later stages of object exploration. Even as infants touch plants less frequently overall than other object types, they particularly avoid touching sharp-looking thorns on both plants and novel artifacts, similar to previous findings demonstrating an avoidance of sharp-angled forms (e.g., Bar \& Neta, 2006; Munar et al., 2015; Silvia \& Barona, 2009). This pattern of results suggests that infants avoid all plants during their initial approach when the uncertainty regarding plant properties is the highest, and then show a more fine-grained avoidance of potentially injurious forms on plants and novel artifacts. This strategy would protect infants from the risk of being hurt initially and prevent further damage during subsequent contact. However, infants in our experiment did not simply avoid pointed shapes in any context: They touched the pointed parts of the familiar artifacts more frequently than the non-pointed parts of those objects. This is consistent with previous work showing that, in the case of manmade artifacts, infants only avoid pointed shapes after having an adverse experience with them (LoBue, 2010b).

It is important to underline that despite arguing that infants possess a behavioral avoidance strategy for plants, we do not claim that avoiding plants entails an affective fear response. Work investigating the physiological correlates of infants' responses to snakes-another ancestrally recurrent danger-found that infants' perceptual sensitivity to snakes was not accompanied by physiological indicators of fear or aversion (Thrasher \& LoBue, 2016). Therefore, fear is not necessarily a reaction to all types of ancestrally recurrent dangers. However, we did not specifically measure affective or autonomic responses in the current study so this question remains to be investigated in future studies.

\subsection{Is the behavioral avoidance strategy about plants per se?}

We argue that infants in the current experiment are exhibiting a behavioral avoidance of plants, but is it possible that infants are responding to some other category of entity? The design of our stimuli allows us to speak to several alternate explanations for our findings. First, infants' avoidance of plants cannot be explained by avoidance of more general shape or color features. The novel artifacts were designed to match to the overall shape of the plants, the shapes of specific plant parts (i.e., leaves), and the green color of the plants. Infants did not avoid these features when they were present in the novel artifacts, but they did avoid the plant stimuli. It is still an open question what combination of features infants use to identify an object as a plant, but our results show that infants' avoidance is not merely a consequence of reacting to specific shape or color features. Second, infants in our study did not respond differently to novel compared to familiar entities more generally, ruling out explanations that infants were simply responding to a broader category of familiar compared to unfamiliar entities. Overall, there is now growing evidence for a plant-specific behavioral avoidance strategy across several studies using a range of different stimuli (Elsner \& Wertz, under revised review; Wertz \& Wynn, 2014a). Nevertheless, it may be that future work uncovers a plausible alternative account of these findings.

\subsection{Limitations and future directions}

The present study has certain limitations. It was conducted in a large urban area with infants whose everyday interactions with plants are limited. It is unclear whether infants and young children who have much richer direct contact with plants and can learn about them from adults who have extensive knowledge about the local vegetation would respond differently. Future cross-cultural work can address this question by assessing the responses to plants in infants and children from modern hunter-gatherer or horticultural societies using a similar experimental design. Additionally, the current study was conducted in a controlled laboratory environment in which infants were seated comfortably on their parent's lap while an adult placed plants and other objects within their reach. Therefore, it is unclear whether we have captured the maximum level of avoidance towards plants that infants may exhibit in real-world environments-if anything, the current study may underestimate the magnitude of the avoidance. An important future direction will be to explore how the behavioral avoidance strategy for plants we observed in the lab operates in more naturalistic settings.

Moreover, the current results reflect infants' baseline responses to plants prior to receiving specific social cues from adults. A critical future step will be to assess infants' responses after they have received clear social information that a plant is harmful to touch. It is well established that infants use information from others to regulate their own behavior (e.g., Baldwin \& Moses, 1996) and that social information plays a crucial role in threat perception (LoBue et al., 2010) and danger learning (e.g., Barrett et al., 2016). Importantly, human adaptations for plants are predicted to include social learning procedures as an integral part of their design (Wertz \& Wynn, 2014a; 2014b). Correspondingly, there is evidence that infants differentially seek out social information when confronted with plants (Elsner \& Wertz, under revised review) and use social information to learn about plants (e.g., learning about plant edibility; Wertz \& Wynn, 2014b).

The current study provided some evidence that social information may impact infants' responses to thorny plants. Specifically, infants whose parents reported prohibiting them from touching plants exhibited fewer touches to thorny plants. Interestingly, there was no correlation between parental prohibition and infants' responses to benign-looking plants. This lack of correlation with parental prohibition and benignlooking plants is consistent with previous results (Elsner \& Wertz, under revised review; Wertz \& Wynn, 2014a), and suggests that social cues from adults may play a particular role in infants' responses 
specifically to thorny plants. Clearly, there is much left to explore about influence of social information on infants' responses to plants.

\subsection{Conclusion}

Our findings add to a growing body of evidence about infants' responses to ancestrally-recurrent threats by demonstrating novel aspects of infants' behavioral avoidance strategy for plants. This strategy serves to mitigate potential harm from plants, whether or not they possess visible indicators of threat, and provides a base from which subsequent social learning processes can operate. Supplementary data to this article can be found online at https://doi.org/10.1016/j.evolhumbehav.2018.06.001.

\section{Declarations of interest}

None.

\section{Acknowledgements}

This work was funded by the Max Planck Society.

\section{References}

Baayen, R. H., Davidson, D. J., \& Bates, D. M. (2008). Mixed-effects modeling with crossed random effects for subjects and items. Journal of Memory and Language, 59(4), 390412.

Bakker, E. S., Gill, J. L., Johnson, C. N., Vera, F. W., Sandom, C. J., Asner, G. P., \& Svenning, J. C. (2016). Combining paleo-data and modern exclosure experiments to assess the impact of megafauna extinctions on woody vegetation. Proceedings of the National Academy of Sciences, 113(4), 847-855.

Baldwin, D. A., \& Moses, L. J. (1996). The ontogeny of social information gathering. Child Development, 67(5), 1915-1939.

Bar, M., \& Neta, M. (2006). Humans prefer curved visual objects. Psychological Science,17(8), 645-648.

Barrett, H.C., \& Broesch, J. (2012). Prepared social learning about dangerous animals in children. Evolution and Human Behavior, 33, 499-508.

Barrett, H. C., Peterson, C. D., \& Frankenhuis, W. E. (2016). Mapping the cultural learnability landscape of danger. Child Development, 87(3), $770-781$.

Bates, D., Mächler, M., Bolker, B., \& Walker, S. (2014). Fitting linear mixed-effects models using Ime4. arXiv preprint arXiv:1406.5823.

Begossi, A., Hanazaki, N., \& Tamashiro, J. Y. (2002). Medicinal plants in the Atlantic Forest (Brazil): knowledge, use, and conservation. Human Ecology, 30(3), 281-299. Broesch, J., Barrett, H. C., \& Henrich, J. (2014). Adaptive content biases in learning about animals across the life course. Human Nature, 25(2), 181-199.

Cashdan, E. (1994). A sensitive period for learning about food. Human Nature, 5(3), 279-291.

Cashdan, E. (1998). Adaptiveness of food learning and food aversions in children. Social Science Information, 37(4), 613-632.

Cooper, S. M., \& Owen-Smith, N. (1986). Effects of plant spinescence on large mammalian herbivores. Oecologia, 68(3), 446-455.

Cordain, L., Miller, J. B., Eaton, S. B., Mann, N., Holt, S. H., \& Speth, J. D. (2000). Plant-animal subsistence ratios and macronutrient energy estimations in worldwide huntergatherer diets. The American Journal of Clinical Nutrition, 71(3), 682-692.

Coss, R. G. (2003). The role of evolved perceptual biases in art and design. In Evolutionary aesthetics (pp. 69-130). Springer, Berlin, Heidelberg.

Crittenden, A. N., Conklin-Brittain, N. L., Marlowe, F. W., Schoeninger, M. J., Wrangham, R.W. (2009). Foraging strategies and diet composition of Hadza children. American Journal of Physical Anthropology, 138(S48): 112.

Elsner, C., \& Wertz, A.E. (under revised review). The seeds of social learning: Infants exhibit more social referencing for plants than other object types. Unpublished results.

Fantz, R. L., \& Miranda, S. B. (1975). Newborn infant attention to form of contour. Child Development, 224-228.

Fox, J. \& Weisberg, S. (2011). An $\{R\}$ Companion to Applied Regression $\left(2^{\text {nd }}\right.$ Ed). Sage: Thousand Oaks CA. Retrieved from http://socserv.socsci.mcmaster.ca/jfox/Books/Companion

Fürstenberg-Hägg, J., Zagrobelny, M., \& Bak, S. (2013). Plant defense against insect herbivores. International Journal of Molecular Sciences, $14(5), 10242-10297$.

Gartstein, M. A., \& Rothbart, M. K. (2003). Studying infant temperament via the revised infant behavior questionnaire. Infant Behavior and Development, 26(1), 64-86.

Gowda, J. H. (1996). Spines of Acacia tortilis: what do they defend and how? Oikos, 279-284.

Greenwald, A. M., Eerkens, J. W., \& Bartelink, E. J. (2016). Stable isotope evidence of juvenile foraging in prehistoric Central California. Journal of Archaeological Science: Reports, 7, 146-154.

Guo, S.X., Sha, J.G., Bian, L.Z., \& Qiu, Y.L. (2009). Male spike strobiles with Gnetum affinity from the Early Cretaceous in western Liaoning, Northeast China. Journal of Systematics and Evolution, 47(2), 93-102.

Gurven, M., Allen-Arave, W., Hill, K., \& Hurtado, M. (2000). "It's a wonderful life": signaling generosity among the Ache of Paraguay. Evolution and Human Behavior,

21(4), 263-282.

Gurven, M., Stieglitz, J., Hooper, P. L., Gomes, C., \& Kaplan, H. (2012). From the womb to the tomb: the role of transfers in shaping the evolved human life history. Experimental Gerontology, 47(10), 807-813.

Hagen, E. H., Sullivan, R. J., Schmidt, R., Morris, G., Kempter, R., \& Hammerstein, P. (2009). Ecology and neurobiology of toxin avoidance and the paradox of drug reward. Neuroscience, 160(1), 69-84.

Halpern, M., Raats, D., \& Lev-Yadun, S. (2007). Plant biological warfare: thorns inject pathogenic bacteria into herbivores. Environmental Microbiology, 9(3), 584-592.

Hardy, K. (2008). Prehistoric string theory. How twisted fibers helped to shape the world. Antiquity, 82(316), 271-280.

Harris, D. R., \& Hillman, G. C. (Eds.). (2014). Foraging and farming: The evolution of plant exploitation (Vol. 31). Routledge.

Hawkes, K., O'Connell, F., \& Jones, N. B. (1995). Hadza children's foraging: juvenile dependency, social arrangements, and mobility among hunter-gatherers. Current Anthropology, 36(4), 688-700.

Henrich, J., Heine, S. J., \& Norenzayan, A. (2010). The weirdest people in the world ? Behavioral and Brain Sciences, 33, 1-23.

Henry, A. G., Brooks, A. S., \& Piperno, D. R. (2014). Plant foods and the dietary ecology of Neanderthals and early modern humans. Journal of Human Evolution, $69,44-54$.

Hess, U., Gryc, O., \& Hareli, S. (2013). How shapes influence social judgments. Social Cognition, 31(1), 72-80.

Hess, U., Gryc, O., \& Hareli, S. (2013). How shapes influence social judgments. Social Cognition, 31(1), 72-80.
Hothorn, T., Bretz, F., Westfall, P. (2008). Multicomp: Simultaneous inference for general linear hypotheses. R Package Version 1.3-6. Retrieved from http:// CRAN.R-project.org

Janzen, D. H. (1986). Chihuahuan desert nopaleras: defaunated big mammal vegetation. Annual Review of Ecology and Systematics, 17(1), 595-636.

Kaplan, H., Hill, K., Lancaster, J., \& Hurtado, A. M. (2000). A theory of human life history evolution: diet, intelligence, and longevity. Evolutionary Anthropology: Issues, News, and Reviews, 9(4), 156-185.

Karban, R. B., \& Baldwin, I. T. (1997) Induced Responses to Herbivory. University of Chicago Press.

Keeler, R.F., \& Tu, A. T. (Eds.). (1983). Handbook of natural toxins: Plant and fungal toxins. (Vol. 1). New York, NY: Marcel Dekker Inc.

Keeling, C. I., \& Bohlmann, J. (2006). Genes, enzymes and chemicals of terpenoid diversity in the constitutive and induced defence of conifers against insects and pathogens. New Phytologist, 170(4), 657-675.

Kingsbury J.M. (1983). The evolutionary and ecological significance of plant toxins. In: Keeler RF, Tu AT, editors. Handbook of natural toxins: Plant and fungal toxins. Vol. 1. New York, NY: Marcel Dekker, Inc; pp. 675-706.

Kortlandt, A. (1980). How might early hominids have defended themselves against large predators and food competitors? Journal of Human Evolution, 9(2), 79-112.

Langenheim, J. H. (1994). Higher plant terpenoids: a phytocentric overview of their ecological roles. Journal of Chemical Ecology, 20(6), 1223-1280.

Larson, C. L., Aronoff, J., \& Stearns, J. J. (2007). The shape of threat: simple geometric forms evoke rapid and sustained capture of attention. Emotion, 7(3), 526.

Lausberg, H., \& Sloetjes, H. (2009). Coding gestural behavior with the NEUROGES-ELAN system. Behavior Research Methods, 41(3), 841-849.

Lee, R. B. (1993). The Dobe Ju/'hoansi (2nd edition). Fort Worth, TX: Harcourt Brace College Publishers.

Lin, J. Y., Arthurs, J., \& Reilly, S. (2017). Conditioned taste aversions: From poisons to pain to drugs of abuse. Psychonomic Bulletin \& Review, 24(2), 335-351.

LoBue, V. (2010a). And along came a spider: An attentional bias for the detection of spiders in young children and adults. Journal of Experimental Child Psychology, 107(1), 5966.

LoBue, V. (2010b). What's so scary about needles and knives? Examining the role of experience in threat detection. Cognition and Emotion, 24(1), $180-187$.

LoBue, V., \& DeLoache, J. S. (2008). Detecting the snake in the grass: Attention to fear-relevant stimuli by adults and young children. Psychological Science, 19(3), 284-289.

LoBue, V., \& DeLoache, J. S. (2010). Superior detection of threat-relevant stimuli in infancy. Developmental Science, 13(1), 221-228.

LoBue, V., Rakison, D. H., \& DeLoache, J. S. (2010). Threat Perception Across the Life Span: Evidence for Multiple Converging Pathways. Current Directions in Psychological Science, 19(6), 375-379.

Munar, E., Gómez-Puerto, G., Call, J., \& Nadal, M. (2015). Common visual preference for curved contours in humans and great apes. PLoS One, 10(11), e0141106.

New, J. J., \& German, T. C. (2015). Spiders at the cocktail party: An ancestral threat that surmounts inattentional blindness. Evolution and Human Behavior, 36(3), 165-173.

Olivier, J., Otto, T., Roddaz, M., Antoine, P. O., Londoño, X., \& Clark, L. G. (2009). First macrofossil evidence of a pre-Holocene thorny bamboo cf. Guadua (Poaceae: Bambusoideae: Bambuseae: Guaduinae) in south-western Amazonia (Madre de Dios-Peru). Review of Palaeobotany and Palynology, 153(1-2), 1-7.

Orrock, J., Connolly, B., \& Kitchen, A. (2017). Induced defences in plants reduce herbivory by increasing cannibalism. Nature Ecology \& Evolution, $1(8)$, 1205.

Öhman, A., Flykt, A., \& Esteves, F. (2001). Emotion drives attention: detecting the snake in the grass. Journal of Experimental Psychology: General, $130(3), 466$.

Palo, R. T., \& Robbins, C. T. (1991). Plant defenses against mammalian herbivory. CRC Press.

Peters, C. R., O'Brien, E. M., Boaz, N. T., Conroy, G. C., Godfrey, L. R., Kawanaka, K., ... \& Smith, E. O. (1981). The early hominid plant-food niche: insights from an analysis of plant exploitation by Homo, Pan, and Papio in eastern and southern Africa [and Comments and Reply]. Current Anthropology, 22(2), 127-140. 
Profet, M. (1992). Pregnancy sickness as adaptation: A deterrent to maternal ingestion of teratogens. In Barkow, J. H., Cosmides, L., \& Tooby, J. (Eds.). The adapted mind: Evolutionary psychology and the generation of culture. Oxford University Press, USA.

Rakison, D. H., \& Derringer, J. (2008). Do infants possess an evolved spider-detection mechanism? Cognition, 107(1), 381-393.

Rakison, D. H., \& Derringer, J. (2008). Do infants possess an evolved spider-detection mechanism? Cognition, 107(1), $381-393$.
Reilly, S., \& Schachtman, T. R. (Eds.). (2008). Conditioned taste aversion: Neural and behavioral processes. Oxford University Press.

Rioux, C., Picard, D., \& Lafraire, J. (2016). Food rejection and the development of food categorization in young children. Cognitive Development, $40,163-177$.

Sikes, N. E. (1994). Early hominid habitat preferences in East Africa: paleosol carbon isotopic evidence. Journal of Human Evolution, 27(1), 25-45.

Silvia, P. J., \& Barona, C. M. (2009). Do people prefer curved objects? Angularity, expertise, and aesthetic preference. Empirical Studies of the Arts, 27(1), 25-42.

Sugiyama, L. S. (2004). Illness, injury, and disability among Shiwiar forager-horticulturalists: Implications of health-risk buffering for the evolution of human life history. American

Journal of Physical Anthropology, 123(4), 371-389.
Team, R. C. (2016). R: A language and environment for statistical computing. Vienna, Austria: R Foundation for Statistical Computing; 2016.

Thrasher, C., \& LoBue, V. (2016). Do infants find snakes aversive? Infants' physiological responses to "fear-relevant" stimuli. Journal of Experimental Child Psychology, 142, $382-390$.

Ungar, P. S., \& Sponheimer, M. (2011). The diets of early hominins. Science, 334(6053), 190-193.

Vartanian, O., Navarrete, G., Chatterjee, A., Fich, L. B., Leder, H., Modroño, C., ... \& Skov, M. (2013). Impact of contour on aesthetic judgments and approach-avoidance decisions in architecture. Proceedings of the National Academy of Sciences, 110(Supplement 2), 10446-10453.

Wertz, A. E., \& Wynn, K. (2014a). Thyme to touch: Infants possess strategies that protect them from dangers posed by plants. Cognition, 130(1), 44-49.

Wertz, A. E., \& Wynn, K. (2014b). Selective social learning of plant edibility in 6-and 18-month-old infants. Psychological Science, 25(4), 874-882.

Westerman, S. J., Gardner, P. H., Sutherland, E. J., White, T., Jordan, K., Watts, D., \& Wells, S. (2012). Product design: Preference for rounded versus angular design elements. Psychology \& Marketing, 29(8), 595-605.
P.

Wiener, A., Shudler, M., Levit, A., \& Niv, M. Y. (2011). BitterDB: a database of bitter compounds. Nucleic Acids Research, 40(D1), D413-D419. 